\title{
Teaching Finite Element Analysis for Design Engineers
}

\author{
Paul M. Kurowski \\ The University of Western Ontario \\ Department of Mechanical and Materials Engineering \\ pkurowski@eng.uwo.ca
}

\begin{abstract}
The Finite Element Analysis (FEA) is becoming increasingly popular among design engineers using it as one of many product design tools. Safe and cost efficient use of FEA as a product design tool requires training, different from that presently found in undergraduate curriculum of mechanical engineering students. The specific requirements of design engineers for training in the field of FEA have been addressed by the author in a number of professional development courses in FEA, catering specifically to the needs of design engineers. This paper discuses tools and methods used in the development and delivery of these courses and their applicability to the undergraduate courses taught in Canadian Engineering schools.
\end{abstract}

\section{Changing responsibilities of design engineers.}

Due to competitive pressures as well as the availability of CAE tools, since early 1990's the responsibilities of design engineer have been steadily expanding from just designing to geometry creation with CAD and design analysis, most often performed with FEA (figure 1).

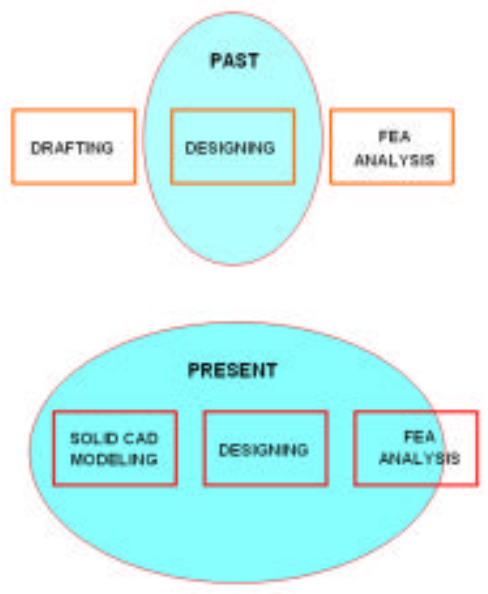

Figure 1. Expanding responsibilities of design engineers
To make sure that FEA performs in the hands of design engineers as a productivity tool, design engineers must be provided with training that addresses the needs of this relatively new type of users.

\section{FEA as a design tool}

Finite Element Analysis (FEA)for a perspective of a design engineer, is one of many tools of design analysis. Therefore, it is appropriate to position it first among other design analysis tools.

Design analysis can be conducted by on real objects or on models that represent certain aspects of the real object. If models are used instead of real objects, the analysis can be conducted earlier in the design process, long before the final product or even prototypes are built. Those models used for design analysis can take the form of physical models for example: scaled down models, mockups, photoelastic models) or mathematical models where certain behavior of a part or a structure is described by a mathematical apparatus.

The design analysis conducted with the use of mathematical models can be further broken down based on what methods are used to obtain solution. 
Simple mathematical models can be solved analytically. More complex models require the use of numerical methods and this is where the FEA comes in. FEA is one of many numerical methods used to solve complex mathematical models. It is particularly well suited to solving structural and thermal problems commonly challenging mechanical design engineers.

\section{What is "FEA for Design Engineers"}

While the expertise expected of FEA users always requires familiarity with Mechanics of Materials, Engineering Design and other topics common to any mechanical engineering curriculum, the FEA training should differentiate between the needs of analysts and the needs of design engineers because each group of uses the FEA differently.

What exactly distinguishes "Finite Element Analysis for Design Engineers" from a "regular" Finite Element Analysis as it traditionally has been performed by analysts? We will highlight the most essential characteristics of FEA for Design Engineers as opposed to FEA performed by analysts.

FEA is just another design tool

For Design Engineers the Finite Element Analysis is one of many design tools and is used along CAD, spreadsheets, catalogs, data bases, hand calculations, text books etc.

FEA is based on CAD models

Design is nowadays almost always created using CAD tools and, therefore, CAD model is the starting point for FEA.

FEA is concurrent with the design process.

Since FEA is a design tool, it should be used concurrently with the design process. It should keep up, or better, drive the design process. Analysis iterations must be preformed fast and, since results are used to make design decisions, the results must be reliable, even though not enough input data may be available for analysis conducted early in the design process.

\section{Limitations of "FEA for Design Engineers"}

As we can see, the FEA used in the design environment should meet quite high requirements. It must be exe cuted fast and accurately; even though it is in the hands of design engineers and not FEA specialists. An obvious question is: would it be better to have a dedicated specialist perform FEA and let design engineers do what they do best: designing new products? The answer depends on the size of organization, type of products, company organization and culture and many other tangible and non tangible factors. A general consensus is that design engineers should handle relatively simple types of analysis in support of design process. More complex types of analyses, too complex and too time consuming to be executed concurrently design process, are usually better handled either by a dedicated analyst or contracted out to specialized consultants.

\section{Objective of "FEA for Design Engineers"}

The ultimate objective of using the FEA as a design tool is to change the design process from iterative cycles of "design, prototype, test" into a streamlined process where prototypes are used only for final design verification. With the use of FEA, design iterations are moved from physical space of prototyping and testing into virtual space of computer based simulations (figure 2). The FEA is not, of course, the only tool of computerized simulation used in the design process. There are others like, for example, Computational Fluid Dynamics, Motion Analysis etc. jointly called the tools of Computer Aided Engineering (CAE).

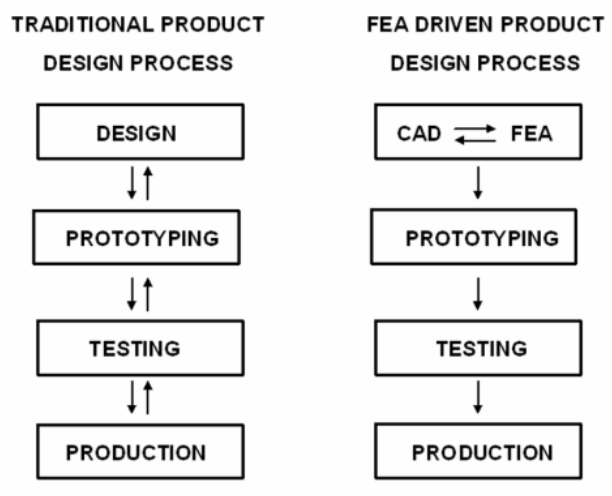
Figure 2. Traditional and CAE driven product
development process

\section{How to teach "FEA for Design Engineers"?}

Teaching an FEA course to engineers who will use it as a design tool requires specific approach not typically found in FEA courses commonly taught to engineering students. It requires a careful balance of FEA fundamentals and examples to illustrate those 
fundamentals while connecting to problems typical to design engineering.

The principal objective of an "FEA for Design Engineers" course is to provide students with skills necessary to perform time efficient and cost-effective FEA concurrently with product design process as well as to manage and supervise in-house FEA activities.

This objective is best achieved by combining lectures with hands-on exercises. Hands-on exercises range from analysis of simple but illustrative models highlighting FEA fundamentals to more complex assignments which can be customized to meet specific needs of students' industry.

Based on this approach the author has developed a number of Professional Development courses covering the use of FEA in the "design engineer" mode. While they differ in length and the software used, the major objectives remain the same:

- To review the fundamentals of the Finite Element Analysis (FEA)

- To provide a basis for assessment of the quality of FEA results

- To demonstrate cost effective modeling techniques

- To conduct different types of analyses typically performed by design engineers

- To provide tools for effective implementation and management of the FEA

- To discuss common traps and misconceptions in the applied FEA

Here we introduce a sample course addressed to design engineers and offered by the Advanced Design and Manufacturing Institute (ADMI) of Canada [1].

\section{ADMI Canada course "Finite Element Analysis for Design Engineers"}

The ADMI Canada course is intended for engineers who wish to use the Finite Element Analysis (FEA) as one of their design tools, and for engineering managers in charge of FEA related projects. It provides the participants with tool necessary to turn FEA into a productivity tool by:

- Understanding powers and shortcomings of FEA

- $\quad$ Avoiding common pitfalls and misconceptions of FEA

- $\quad$ Selecting software best suited for the analyzed products
- $\quad$ Selecting preferable modeling approaches

- $\quad$ Producing reliable results on time

- Integrating FEA with CAD and other CAE tools

- Streamlining CAD and FEA by implementing FEA oriented Solid Modeling practices

- Ensuring quality and cost-effectiveness of inhouse and contracted out FEA projects

The ADMI Canada course has a modular structure typically spanning over two four days blocks:

\section{MODULE 1}

Day 1

- Position of FEA among other tools of numerical analysis

- Design and types of finite elements

- Controlling discretization errors

- $\quad h$ convergence process

- $\quad$ p convergence process

Day 2

- Finite element mesh

- Mesh compatibility

- Common meshing problems

- Modeling process

- Common modeling techniques

- $\quad$ Test \#1 5\%

Day 3

- Common modeling techniques

- $\quad$ Types of finite element analysis

- $\quad$ Steady state thermal analysis

- Transient thermal analysis

- Thermal stress analysis

Day 4

- Modal analysis

- Linear buckling analysis

- Nonlinear buckling analysis

- Test \#2 5\%

IN BETWEEN MODULES

- Two take home assignments $25 \%$ each

\section{MODULE 2}

Day 1

- Nonlinear material analysis

- Nonlinear geometry analysis

- $\quad$ Test \#3 5\%

Day 2

- Modal analysis ... cont'd

- Vibration analysis 
- $\quad$ Interfacing FEA with Motion Analysis software

- $\quad$ Interfacing FEA with CFD tools

Day 3

- Preparing CAD geometry for FEA

- CAD geometry quality

- $\quad$ Test \#4 5\%

$\underline{\text { Day } 4}$

- CAD data exchange between different CAD and FEA systems

- Implementation of FEA into design process

- FEA project management

- Common FEA traps and misconceptions

- $\quad$ Final exam $\quad 30 \%$

Almost all topics are illustrated by hands-on examples using a commercial FEA software (here COSMOSWorks), however, the course is not software specific and the acquired skills are applicable to any FEA software.

\section{How to select FEA software for "FEA foe Design Engineers"?}

Considering that design engineers typically do not create model specifically for analysis but use CAD models both for design and analysis, the FEA software used in the course must be integrated with CAD.

CAD and FEA software must, therefore, satisfy the following requirements.

\section{$\underline{\text { CAD system should }}$}

- create $100 \%$ of complex geometry:

- $\quad$ both CAD specific and FEA specific

- offer the ability of quick switches between

- those two geometries while keeping them linked

- $\quad$ offer close integration with FEA

\section{$\underline{\text { FEA system should }}$}

- $\quad$ mesh relevant CAD produced geometry automatically

- user's intervention should not be required

- map finite elements precisely to geometry

- offer optimization and sensitivity studies capabilities

- $\quad$ offer close integration with CAD

\section{Sample problems}

All problems solved by students hand-on illustrate and reinforce the discussed topics. Simple models are used in class to speed up the model definition and the solution process and to focus problems illustrated by each one. More complex problems are solved in takehome assignments.

We will review typical in-class problems. The tensile hollow strip model is used to demonstrate the discretization error and the convergence process (figure 3).

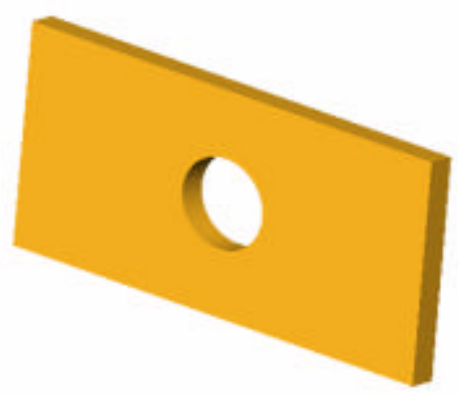

Figure 3. Hollow tensile strip

The analysis of L shape bracket (figure 4) model illustrates divergent solution due to stress singularity related to the error in the formulation of the mathematical model.

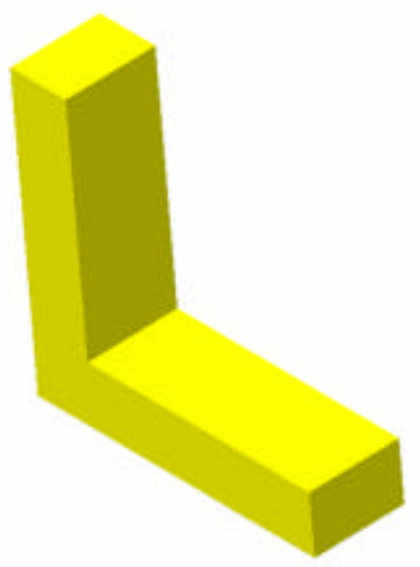

Figure 4. L shape bracket with sharp reentrant corner 
The stamped steel pulley (figure 5) exercise is used to discuss the use of shell elements, the symmetry boundary conditions and the limited capabilities of the FEA to model stresses in accordance with the mechanics of materials.

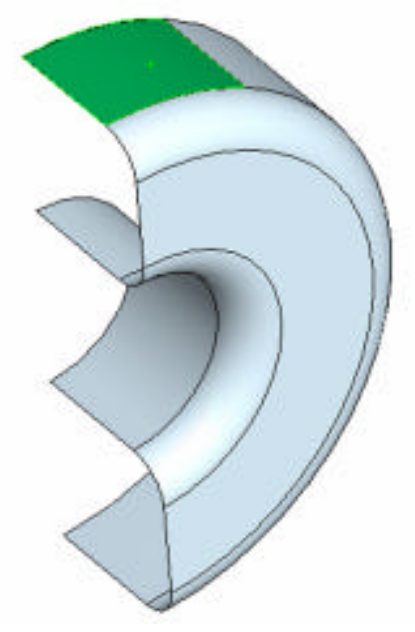

Figure 5. Stamped steel pulley

The tuning fork (figure 6) is used for modal analysis to demonstrate he effect of supports on the modes of vibration and the nature of modes of vibration in a symmetric model .

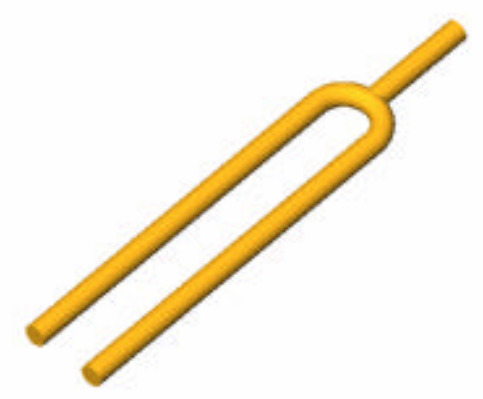

Figure 6. Tuning fork

The heat sink (figure 7) example introduces steady state and transient thermal analysis considering the effects of convection.

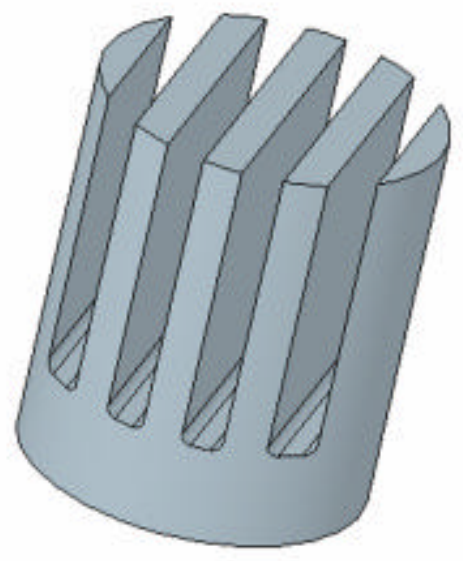

Figure 7. Heat sink

Take home assignments offer the students opportunities to work on more complex models of parts or assemblies which may be chosen based on individual interests. For example, students are asked to select a part or a subassembly of a car (figure 8) for a complete structural analysis which includes formulation of loads, restraints and all required modeling assumptions.

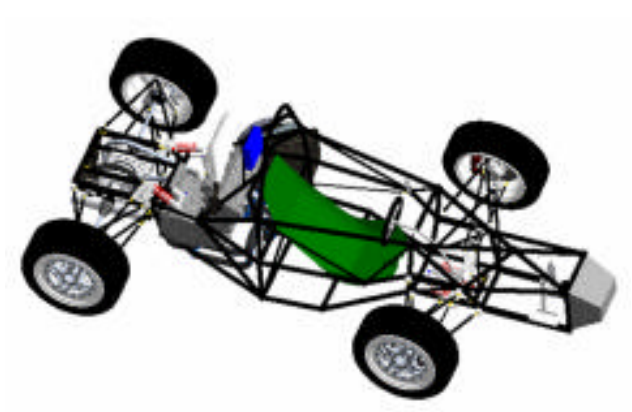

Figure 8. SAE formula car model

\section{FEA Implementation and Management}

An important part of "FEA for Design Engineers" is implementation of FEA in the design engineering environment and management of FEA projects.

It familiarizes students with steps typical to any FEA project, report writing and project documentation as well as with traps and misconceptions often found in everyday industrial applications of FEA. 
"FEA Implementation and Management" section of the course discusses project check lists (figure 9) and errors commonly found in FEA projects. It proposes

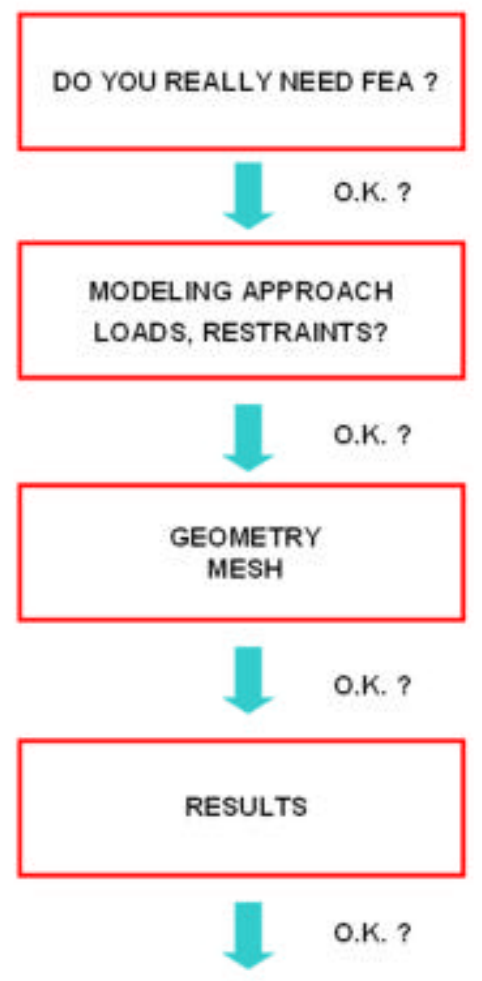

Figure 9. A sample FEA project check list

This section is of particular interest to engineering managers who may never work with FEA "hands-on" but assume responsibility for design decisions made using FEA results.

\section{9. "FEA for Design Engineers" as an undergraduate course}

The approach to teaching FEA as a design tool has been discussed here using the ADMI Canada course as an example. Similar approach has been used successfully in the development of other Professional Development courses as well as in text books and other technical publications in field of FEA [2], [3], [4].

This combined experience makes the author convinced that the same approach may be used in a successful delivery of an undergraduate course in a mechanical engineering program.
While "FEA for Design Engineers" course should not replace standard FEA courses typically offered to undergraduate Mechanical Engineering students, it would complement them very well by providing students with readily marketable skills in numerical design analysis.

"FEA for Design Engineers" could be offered as a "stand-alone" half course, best placed in the third or fourth year or could be combined with standard FEA half course into a full year course.

\section{References}

[1] www.admicanada.com/courses/

[2] P. M. Kurowski, "Finite Element Analysis for Design Engineers", the Society of Automotive Engineers, 2004.

[3] P. M. Kurowski, "Engineering Analysis with COSMOSWorks", Schroff Development Corporation, 2006

[4] P. M. Kurowski "Going Mainstream Analysis", SolidWorks Corporation, 2004 